\title{
EXPLICIT EXPONENTIAL RUNGE-KUTTA METHODS FOR SEMILINEAR PARABOLIC PROBLEMS
}

\author{
MARLIS HOCHBRUCK* ${ }^{*}$ AND ALEXANDER OSTERMANN**,
}

\begin{abstract}
The aim of this paper is to analyze explicit exponential Runge-Kutta methods for the time integration of semilinear parabolic problems. The analysis is performed in an abstract Banach space framework of sectorial operators and locally Lipschitz continuous nonlinearities. We commence by giving a new and short derivation of the classical (nonstiff) order conditions for exponential RungeKutta methods, but the main interest of our paper lies in the stiff case. By expanding the errors of the numerical method in terms of the solution, we derive new order conditions that form the basis of our error bounds for parabolic problems. We show convergence for methods up to order four and we analyze methods that were recently presented in the literature. These methods have classical order four, but they do not satisfy some of the new conditions. Therefore, an order reduction is expected. We present numerical experiments which show that this order reduction in fact arises in practical examples. Based on our new conditions, we finally construct methods that do not suffer from order reduction.
\end{abstract}

1. Introduction. Motivated by recent interest in exponential integrators for stiff problems $[3,9,11,12]$ and inspired by the promising numerical experiments reported in these papers, we present error bounds for a class of explicit exponential Runge-Kutta methods for semilinear parabolic problems

$$
u^{\prime}(t)+A u(t)=g(t, u(t)), \quad u\left(t_{0}\right)=u_{0} .
$$

The idea behind exponential integrators is an old one and dates back to the sixties of the past century. The early literature on exponential one-step methods comprises $[4,6,14,23,24,25]$. In most of these papers, the methods were constructed by making use of the variation-of-constants formula for the solution of (1.1). All of them use the exponential function of the matrix $-h A$ in order to step from time $t$ to time $t+h$, or some rational approximations thereof. Exponential multistep methods have been considered in $[13,21,26]$.

Although the first exponential integrators were proposed many years ago, such methods have not been regarded as practical for a long time. This view, however, has changed recently as new methods for computing or approximating the product of a matrix exponential function with a vector have been developed, see the review [19] and references therein. For large problems, polynomial approximations have to be applied, in general, either based on Chebyshev polynomials or using variants of the Lanczos process or the Arnoldi method. These techniques are also reviewed in [19]. For parabolic problems it has been shown recently that one can achieve grid independent convergence of the Lanczos process by working with a shifted and inverted matrix $[5,20]$. The linear systems arising in each step of the Lanczos process can either be solved directly (if fast direct solvers are available) or with a preconditioned iterative method. Details can be found in [5].

The numerical comparisons presented in $[11,12,18]$ reveal a number of examples where explicit exponential integrators outperform standard integrators. However,

*Mathematisches Institut, Heinrich-Heine Universität Düsseldorf, Universitätsstr. 1, D-40225 Düsseldorf, Germany (E-mail: marlis@am.uni-duesseldorf.de)

**Institut für Mathematik, Universität Innsbruck, Technikerstr. 13, A-6020 Innsbruck, Austria (E-mail: alexander.ostermann@uibk.ac.at)

${ }^{\ddagger}$ Corresponding author.

$\ddagger \ddagger$ Version of December 16, 2004. 
while the convergence behavior of implicit or linearly implicit Runge-Kutta or multistep methods for parabolic problems is nowadays well understood $[15,16]$, no analysis is known so far for explicit exponential integrators. In our paper [10] we have analyzed implicit exponential Runge-Kutta methods. There, a crucial step in the convergence proofs was to show that all stages have a sufficiently small defect when the true solution is inserted in place of the numerical solution. This is no longer true for explicit schemes of order at least three. Therefore, new techniques have to be used for proving error bounds in this case. Our aim with this paper is to derive new order conditions for stiff problems and based on these to give error bounds for parabolic problems. The new conditions will then enable us to analyze the methods presented in the literature and further to construct new methods that do not suffer from order reduction.

The outline of the paper is as follows: In Section 2, we define a general class of exponential Runge-Kutta methods and give conditions under which these methods preserve equilibria. We further give a simple and short derivation of the classical order conditions for arbitrary order. Our convergence analysis of (1.1) will be performed in the standard framework of analytic semigroups and locally Lipschitz continuous nonlinearities in a Banach space. This abstract framework is recalled in Section 3. Our main results are contained in Section 4, where we derive new order conditions for explicit exponential Runge-Kutta methods applied to parabolic problems. These conditions which comprise the classical order conditions are given in Table 4.1 up to order four. Based on them, we show convergence for explicit exponential RungeKutta methods up to order four, under appropriate temporal smoothness of the exact solution. The convergence results for the exponential Euler method and for secondorder methods are given in Theorems 4.2 and 4.3, respectively, our main result is Theorem 4.7. The new order conditions are further used in Section 5 to analyze methods from the literature and to construct new methods. In particular, we will show that neither of the exponential classical Runge-Kutta methods from $[3,12]$ is of order four, in general, when applied to parabolic problems. Our order conditions enable us, however, to construct a new exponential variant of the classical Runge-Kutta method which is of full order four. In Section 6 we present numerical experiments which show that the order reductions predicted by our theory may in fact arise in practical examples.

2. Order conditions and general properties. We consider the following general class of one-step methods

$$
\begin{aligned}
u_{n+1} & =\chi(-h A) u_{n}+h \sum_{i=1}^{s} b_{i}(-h A) G_{n i}, \\
U_{n i} & =\chi_{i}(-h A) u_{n}+h \sum_{j=1}^{s} a_{i j}(-h A) G_{n j}, \\
G_{n j} & =g\left(t_{n}+c_{j} h, U_{n j}\right)
\end{aligned}
$$

for solving (1.1). Here, the method coefficients $\chi, \chi_{i}, a_{i j}$, and $b_{i}$ are constructed from exponential functions or rational approximations of such functions evaluated at the matrix or operator $-h A$.

For consistency reasons, we always assume that $\chi(0)=\chi_{i}(0)=1$. It seems worth mentioning that (2.1) reduces to a Runge-Kutta method with coefficients $b_{i}=$ $b_{i}(0)$ and $a_{i j}=a_{i j}(0)$ if we consider the limit $A \rightarrow 0$. The latter method will be called underlying Runge-Kutta method henceforth, while (2.1) will be referred to as 
an exponential Runge-Kutta method in the following. We suppose throughout the paper that the underlying Runge-Kutta method satisfies

$$
\sum_{j=1}^{s} b_{j}(0)=1, \quad \sum_{j=1}^{s} a_{i j}(0)=c_{i}, \quad i=1, \ldots, s,
$$

which makes it invariant under the transformation of (1.1) to autonomous form.

A desirable property of numerical methods is that they preserve equilibria $u^{\star}$ of the autonomous problem $u^{\prime}(t)+A u(t)=g(u(t))$. Requiring $U_{n i}=u_{n}=u^{\star}$ for all $i$ and $n \geq 0$ immediately yields the necessary and sufficient conditions. It turns out that the coefficients of the method have to satisfy

$$
\sum_{j=1}^{s} b_{j}(z)=\frac{\chi(z)-1}{z}, \quad \sum_{j=1}^{s} a_{i j}(z)=\frac{\chi_{i}(z)-1}{z}, \quad i=1, \ldots, s .
$$

Without further mentioning, we will assume throughout the paper that these conditions are fulfilled.

With the help of (2.2), the functions $\chi$ and $\chi_{i}$ can be eliminated in (2.1). The numerical scheme then takes the form

$$
\begin{aligned}
u_{n+1} & =u_{n}+h \sum_{i=1}^{s} b_{i}(-h A)\left(G_{n i}-A u_{n}\right), \\
U_{n i} & =u_{n}+h \sum_{j=1}^{s} a_{i j}(-h A)\left(G_{n j}-A u_{n}\right) .
\end{aligned}
$$

Conditions (2.2) also imply that we can restrict ourselves to autonomous problems

$$
u^{\prime}(t)+A u(t)=g(u(t)), \quad u\left(t_{0}\right)=u_{0},
$$

since all methods satisfying (2.2) are invariant under the transformation of (1.1) to autonomous form.

For $A=0$, the methods reduce to classical Runge-Kutta methods, for which the order conditions are well-known. In the case of $A \neq 0$, nonstiff order conditions of order up to 5 have been presented by Friedli [6] using Taylor series expansion of the exact and the numerical solutions, see also [25] and references therein. Our approach below is based on trees which allows to extract the order conditions for arbitrary orders in a systematic and simple way, very similar to classical Runge-Kutta methods. A more technical approach based on the theory of B-series is chosen in [1].

Writing (2.4) in the form

$$
u^{\prime}=g(u)-A u=F(u)
$$

shows that the Taylor expansion of the exact solution contains the elementary differentials of $F$ which are represented by the usual trees. Note that these differentials coincide with those of $g$ except for $F^{\prime}=g^{\prime}-A$ and $F$ itself. Splitting the trees that contain $F^{\prime}$, we obtain trees with two kinds of nodes. The nodes corresponding to $A$ are represented by open (white) circles, whereas the evaluation of $F$ and of derivatives of $g$ is represented by filled (black) circles. This gives the subclass of bi-colored trees with black branching nodes and black leafs. 


\begin{tabular}{|c|c|c|c|c|}
\hline No. & tree & order & differential & order condition \\
\hline 1 & & 1 & $F$ & $\sum \beta_{i}^{(0)}=1$ \\
\hline 2 & & 2 & $g^{\prime} F$ & $\sum \beta_{i}^{(0)} \alpha_{i j}^{(0)}=\frac{1}{2}$ \\
\hline 3 & & 2 & $A F$ & $\sum \beta_{i}^{(1)}=\frac{1}{2}$ \\
\hline 4 & & 3 & $g^{\prime \prime}(F, F)$ & $\sum \beta_{i}^{(0)} \alpha_{i j}^{(0)} \alpha_{i k}^{(0)}=\frac{1}{3}$ \\
\hline 5 & & 3 & $g^{\prime} g^{\prime} F$ & $\sum \beta_{i}^{(0)} \alpha_{i j}^{(0)} \alpha_{j k}^{(0)}=\frac{1}{6}$ \\
\hline 6 & & 3 & $g^{\prime} A F$ & $\sum \beta_{i}^{(0)} \alpha_{i j}^{(1)}=\frac{1}{6}$ \\
\hline 7 & & 3 & $A g^{\prime} F$ & $\sum \beta_{i}^{(1)} \alpha_{i j}^{(0)}=\frac{1}{6}$ \\
\hline 8 & & 3 & $A A F$ & $\sum \beta_{i}^{(2)}=\frac{1}{6}$ \\
\hline 9 & & 4 & $g^{\prime \prime \prime}(F, F, F)$ & $\sum \beta_{i}^{(0)} \alpha_{i j}^{(0)} \alpha_{i k}^{(0)} \alpha_{i l}^{(0)}=\frac{1}{4}$ \\
\hline 10 & & 4 & $g^{\prime \prime}\left(g^{\prime} F, F\right)$ & $\sum \beta_{i}^{(0)} \alpha_{i j}^{(0)} \alpha_{i k}^{(0)} \alpha_{k l}^{(0)}=\frac{1}{8}$ \\
\hline 11 & & 4 & $g^{\prime \prime}(A F, F)$ & $\sum \beta_{i}^{(0)} \alpha_{i j}^{(0)} \alpha_{i k}^{(1)}=\frac{1}{8}$ \\
\hline 12 & & 4 & $g^{\prime} g^{\prime \prime}(F, F)$ & $\sum \beta_{i}^{(0)} \alpha_{i j}^{(0)} \alpha_{j k}^{(0)} \alpha_{j l}^{(0)}=\frac{1}{12}$ \\
\hline 13 & & 4 & $A g^{\prime \prime}(F, F)$ & $\sum \beta_{i}^{(1)} \alpha_{i j}^{(0)} \alpha_{i k}^{(0)}=\frac{1}{12}$ \\
\hline 14 & & 4 & $g^{\prime} g^{\prime} g^{\prime} F$ & $\sum \beta_{i}^{(0)} \alpha_{i j}^{(0)} \alpha_{j k}^{(0)} \alpha_{k l}^{(0)}=\frac{1}{24}$ \\
\hline 15 & & 4 & $g^{\prime} g^{\prime} A F$ & $\sum \beta_{i}^{(0)} \alpha_{i j}^{(0)} \alpha_{j k}^{(1)}=\frac{1}{24}$ \\
\hline 16 & & 4 & $g^{\prime} A g^{\prime} F$ & $\sum \beta_{i}^{(0)} \alpha_{i j}^{(1)} \alpha_{j k}^{(0)}=\frac{1}{24}$ \\
\hline 17 & & 4 & $A g^{\prime} g^{\prime} F$ & $\sum \beta_{i}^{(1)} \alpha_{i j}^{(0)} \alpha_{j k}^{(0)}=\frac{1}{24}$ \\
\hline 18 & & 4 & $g^{\prime} A A F$ & $\sum \beta_{i}^{(0)} \alpha_{i j}^{(2)}=\frac{1}{24}$ \\
\hline 19 & & 4 & $A g^{\prime} A F$ & $\sum \beta_{i}^{(1)} \alpha_{i j}^{(1)}=\frac{1}{24}$ \\
\hline 20 & & 4 & $A A g^{\prime} F$ & $\sum \beta_{i}^{(2)} \alpha_{i j}^{(0)}=\frac{1}{24}$ \\
\hline 21 & & 4 & $A A A F$ & $\sum \beta_{i}^{(3)}=\frac{1}{24}$ \\
\hline
\end{tabular}

TABLE 2.1

Order trees and nonstiff order conditions for exponential Runge-Kutta methods 
For the numerical scheme written in the form $(2.3)$, we define $\widetilde{F}(u)=g(u)-A u_{n}$. Then, (2.3) can be formally interpreted as an ordinary Runge-Kutta method with the usual trees corresponding to the elementary differentials of $\widetilde{F}$. The differentials of $\widetilde{F}$ coincide with those of $g$ except for the evaluation of the functions themselves where $F\left(u_{n}\right)=\widetilde{F}\left(u_{n}\right)$. Moreover, taking the series expansion of the coefficients $a_{i j}$ and $b_{i}$ into account leads to the same bi-colored trees as for the exact solution.

The derivation of the order conditions from the trees is as follows. For sake of simplicity in presentation, we formally define

$$
a_{i j}(z)=\sum_{k \geq 0} \alpha_{i j}^{(k)} z^{k}, \quad b_{i}(z)=\sum_{k \geq 0} \beta_{i}^{(k)} z^{k} .
$$

A black node preceded by another black node is interpreted as $\alpha_{i j}^{(0)}$ whereas a black root is interpreted as $\beta_{i}^{(0)}$. This is exactly the same interpretation as for the underlying Runge-Kutta method. Moreover, $k$ subsequent white nodes followed by a black node are interpreted as $\beta_{i}^{(k)}$ or $\alpha_{i j}^{(k)}$, respectively, depending on whether they appear at the root or not, respectively. This is seen directly from (2.5) with $z=-h A$. The order conditions up to order 4 are displayed in Table 2.1.

3. Analytical framework. Our analysis below will be based on an abstract formulation of (1.1) as an evolution equation in a Banach space $(X,\|\cdot\|)$. Let $\mathcal{D}(A)$ denote the domain of $A$ in $X$. Our basic assumptions on the operator $A$ are that of $[8]$.

Assumption 1. Let $A: \mathcal{D}(A) \rightarrow X$ be sectorial, i.e. $A$ is a densely defined and closed linear operator on $X$ satisfying the resolvent condition

$$
\left\|(\lambda I-A)^{-1}\right\|_{X \leftarrow X} \leq \frac{M}{|\lambda-a|}
$$

on the sector $\{\lambda \in \mathbb{C}: \vartheta \leq|\arg (\lambda-a)| \leq \pi, \lambda \neq a\}$ for $M \geq 1, a \in \mathbb{R}$, and $0<\vartheta<\pi / 2$.

Under this assumption, the operator $-A$ is the infinitesimal generator of an analytic semigroup $\left\{\mathrm{e}^{-t A}\right\}_{t \geq 0}$. For $\omega>-a$, the fractional powers of $\widetilde{A}=A+\omega I$ are well-defined. The following stability bounds are proved in [10]. They are crucial in our analysis.

LEMma 3.1. Under Assumption 1 and for fixed $\omega>-a$, the following bounds hold uniformly on $0 \leq t \leq T$

$$
\begin{gathered}
\left\|\mathrm{e}^{-t A}\right\|_{X \leftarrow X}+\left\|t^{\gamma} \widetilde{A}^{\gamma} \mathrm{e}^{-t A}\right\|_{X \leftarrow X} \leq C, \quad \gamma \geq 0, \\
\left\|h A \sum_{j=1}^{n-1} \mathrm{e}^{-j h A}\right\|_{X \leftarrow X} \leq C .
\end{gathered}
$$

The next lemma (often called Abel's partial summation) is a discrete version of the integration-by-parts formula. Its proof is straightforward.

LEMMA 3.2. For $W_{k}=\sum_{j=0}^{k} w_{j}$ the following summation-by-parts formula holds

$$
\sum_{j=0}^{n-1} w_{j} v_{n-j}=W_{n-1} v_{1}-\sum_{j=0}^{n-2} W_{j}\left(v_{n-j-1}-v_{n-j}\right) .
$$


The stability estimate (3.2a) enables us to define the bounded operators

$$
\varphi_{j}(-t A)=\frac{1}{t^{j}} \int_{0}^{t} \mathrm{e}^{-(t-\tau) A} \frac{\tau^{j-1}}{(j-1) !} \mathrm{d} \tau, \quad j \geq 1 .
$$

We note for later use that $\varphi_{0}(z)=\mathrm{e}^{z}$ and

$$
\varphi_{k+1}(z)=\frac{\varphi_{k}(z)-1 / k !}{z}, \quad \varphi_{k}(0)=\frac{1}{k !}, \quad k \geq 0 .
$$

Our basic assumptions on $g$ are that of [8] and [22]. We thus choose $0 \leq \alpha<1$ and define $V=\mathcal{D}\left(\widetilde{A}^{\alpha}\right) \subset X$ where $\widetilde{A}$ denotes the shifted operator $\widetilde{A}=A+\omega I$ with $\omega>-a$. The linear space $V$ is a Banach space with norm $\|v\|_{V}=\left\|\widetilde{A}^{\alpha} v\right\|$. Note that $V$ does not depend on $\omega$, since different choices of $\omega$ lead to equivalent norms. Our main hypothesis on the nonlinearity $g$ is the following.

Assumption 2. Let $g:[0, T] \times V \rightarrow X$ be locally Lipschitz-continuous in a strip along the exact solution $u$. Thus there exists a real number $L(R, T)$ such that

$$
\|g(t, v)-g(t, w)\| \leq L\|v-w\|_{V}
$$

for all $t \in[0, T]$ and $\max \left(\|v-u(t)\|_{V},\|w-u(t)\|_{V}\right) \leq R$.

EXAMPLE. It is well known that reaction-diffusion equations fit in this abstract framework, as well as the incompressible Navier-Stokes equations in two and three space dimensions, see e.g., [8, Chapter 3] and [17, Section 7.3].

For high-order convergence results, we have to assume more regularity.

Assumption 3. We suppose that (1.1) possesses a sufficiently smooth solution $u:[0, T] \rightarrow V$ with derivatives in $V$, and that $g:[0, T] \times V \rightarrow X$ is sufficiently often Fréchet differentiable in a strip along the exact solution. All occurring derivatives are supposed to be uniformly bounded.

Note that, under the above assumption, the composition

$$
f:[0, T] \rightarrow X: t \mapsto f(t)=g(t, u(t))
$$

is a smooth mapping, too. This will be used frequently.

4. Convergence results for exponential methods. In this section, we discuss the exponential counterparts of classical Runge-Kutta methods and study their convergence properties for the semilinear problem (1.1). More precisely, we consider methods with

$$
\chi(z)=\mathrm{e}^{z} \quad \text { and } \quad \chi_{i}(z)=\mathrm{e}^{c_{i} z}, \quad 1 \leq i \leq s .
$$

Our main interest lies in explicit methods for which, due to $c_{1}=0$,

$$
\chi_{1}(z)=1 \quad \text { and } \quad a_{i j}(z)=0, \quad 1 \leq i \leq j \leq s .
$$

Implicit methods have been analyzed in detail in our recent paper [10]. The scheme (2.1) satisfying (4.1) and (4.2) is called an explicit exponential Runge-Kutta method henceforth. For the coefficients of the exponential Runge-Kutta method (2.1) we assume stability assumptions similar to $(3.2 \mathrm{a})$

$$
\|\phi(-t A)\|_{X \leftarrow X}+\left\|t^{\gamma} \tilde{A}^{\gamma} \phi(-t A)\right\|_{X \leftarrow X} \leq C, \quad 0 \leq \gamma \leq 1
$$

for $\phi=b_{i}$ or $\phi=a_{i j}, i, j=1, \ldots, s$. This assumption is satisfied by all methods considered below, since $b_{i}(z)$ and $a_{i j}(z)$ are (linear) combinations of the functions $\varphi_{k}(z)$ and $\varphi_{k}\left(c_{l} z\right)$, respectively. 
4.1. Error recursion and representation of the defects. In order to simplify the notation, we set again $f(t)=g(t, u(t))$. Our proofs are heavily based on the representation of the exact solution by the variation-of-constants formula

$$
u\left(t_{n}+\theta h\right)=\mathrm{e}^{-\theta h A} u\left(t_{n}\right)+\int_{0}^{\theta h} \mathrm{e}^{-(\theta h-\tau) A} f\left(t_{n}+\tau\right) \mathrm{d} \tau
$$

We expand $f$ into a Taylor series with remainder in integral form to get

$$
f\left(t_{n}+\tau\right)=\sum_{j=1}^{q} \frac{\tau^{j-1}}{(j-1) !} f^{(j-1)}\left(t_{n}\right)+\int_{0}^{\tau} \frac{(\tau-\sigma)^{q-1}}{(q-1) !} f^{(q)}\left(t_{n}+\sigma\right) \mathrm{d} \sigma .
$$

On the one hand, inserting this into the right-hand side of (4.4) yields

$$
\begin{aligned}
u\left(t_{n}+c_{i} h\right)= & \mathrm{e}^{-c_{i} h A} u\left(t_{n}\right)+\sum_{j=1}^{q_{i}}\left(c_{i} h\right)^{j} \varphi_{j}\left(-c_{i} h A\right) f^{(j-1)}\left(t_{n}\right) \\
& +\int_{0}^{c_{i} h} \mathrm{e}^{-\left(c_{i} h-\tau\right) A} \int_{0}^{\tau} \frac{(\tau-\sigma)^{q_{i}-1}}{\left(q_{i}-1\right) !} f^{\left(q_{i}\right)}\left(t_{n}+\sigma\right) \mathrm{d} \sigma \mathrm{d} \tau .
\end{aligned}
$$

On the other hand, inserting the exact solution into the numerical scheme gives

$$
\begin{gathered}
u\left(t_{n}+c_{i} h\right)=\mathrm{e}^{-c_{i} h A} u\left(t_{n}\right)+h \sum_{j=1}^{i-1} a_{i j}(-h A) f\left(t_{n}+c_{j} h\right)+\Delta_{n i}, \\
u\left(t_{n+1}\right)=\mathrm{e}^{-h A} u\left(t_{n}\right)+h \sum_{i=1}^{s} b_{i}(-h A) f\left(t_{n}+c_{i} h\right)+\delta_{n+1}
\end{gathered}
$$

with defects $\Delta_{n i}$ and $\delta_{n+1}$. Substituting (4.5) into (4.7a) we obtain

$$
\begin{aligned}
u\left(t_{n}+c_{i} h\right)= & \mathrm{e}^{-c_{i} h A} u\left(t_{n}\right)+h \sum_{k=1}^{i-1} a_{i k}(-h A) \sum_{j=1}^{q_{i}} \frac{\left(c_{k} h\right)^{j-1}}{(j-1) !} f^{(j-1)}\left(t_{n}\right) \\
& +h \sum_{k=1}^{i-1} a_{i k}(-h A) \int_{0}^{c_{k} h} \frac{\left(c_{k} h-\sigma\right)^{q_{i}-1}}{\left(q_{i}-1\right) !} f^{\left(q_{i}\right)}\left(t_{n}+\sigma\right) \mathrm{d} \sigma+\Delta_{n i} .
\end{aligned}
$$

Subtracting (4.6) from (4.8) gives the following explicit representation of the defects

$$
\Delta_{n i}=\sum_{j=1}^{q_{i}} h^{j} \psi_{j, i}(-h A) f^{(j-1)}\left(t_{n}\right)+\Delta_{n i}^{\left[q_{i}\right]}
$$

with remainders $\Delta_{n i}^{\left[q_{i}\right]}$, and with

$$
\psi_{j, i}(-h A)=\varphi_{j}\left(-c_{i} h A\right) c_{i}^{j}-\sum_{k=1}^{i-1} a_{i k}(-h A) \frac{c_{k}^{j-1}}{(j-1) !} .
$$

Similarly, we get for the defects at time $t_{n+1}$

$$
\delta_{n+1}=\sum_{j=1}^{q} h^{j} \psi_{j}(-h A) f^{(j-1)}\left(t_{n}\right)+\delta_{n+1}^{[q]},
$$


where

$$
\psi_{j}(-h A)=\varphi_{j}(-h A)-\sum_{k=1}^{s} b_{k}(-h A) \frac{c_{k}^{j-1}}{(j-1) !}
$$

For the remainders in (4.9) and (4.11), we have the following estimate.

Lemma 4.1. Let $0<\nu \leq 1$ and $\widetilde{A}^{\nu-1} f^{(r)} \in L^{\infty}(0, T ; V)$. Then, it holds

$$
\begin{gathered}
h^{1-\nu}\left\|\Delta_{n i}^{[r]}\right\|_{V}+\left\|\widetilde{A}^{\nu-1} \Delta_{n i}^{[r]}\right\|_{V} \leq C h^{r+1} \sup _{0 \leq \tau \leq 1}\left\|\widetilde{A}^{\nu-1} f^{(r)}\left(t_{n}+\tau h\right)\right\|_{V}, \\
\left\|\sum_{j=0}^{n-1} \mathrm{e}^{-j h A} \delta_{n-j}^{[r]}\right\|_{V} \leq C h^{r} \sup _{0 \leq t \leq t_{n}}\left\|\widetilde{A}^{\nu-1} f^{(r)}(t)\right\|_{V}
\end{gathered}
$$

with a constant $C$, uniformly in $0 \leq t_{n} \leq T$.

Proof. Both estimates follow at once from the stability bound

$$
\left\|t^{\gamma} \widetilde{A}^{\gamma} \mathrm{e}^{-t A}\right\|_{V \leftarrow V}+\left\|t^{\gamma} \widetilde{A}^{\gamma} \phi(-t A)\right\|_{V \leftarrow V} \leq C, \quad 0 \leq \gamma \leq 1
$$

for $\phi=b_{i}$ or $\phi=a_{i j}, i, j=1, \ldots, s$. The latter is a consequence of (3.2a) and (4.3), respectively.

Let $e_{n}=u_{n}-u\left(t_{n}\right)$ and $E_{n i}=U_{n i}-u\left(t_{n}+c_{i} h\right)$ denote the differences between the numerical and the exact solutions. Subtracting (4.7) from the numerical method (2.1) satisfying (4.1) and (4.2) gives the error recursion

$$
\begin{gathered}
E_{n i}=\mathrm{e}^{-c_{i} h A} e_{n}+h \sum_{j=1}^{i-1} a_{i j}(-h A)\left(g\left(t_{n}+c_{j} h, U_{n j}\right)-f\left(t_{n}+c_{j} h\right)\right)-\Delta_{n i}, \\
e_{n+1}=\mathrm{e}^{-h A} e_{n}+h \sum_{i=1}^{s} b_{i}(-h A)\left(g\left(t_{n}+c_{i} h, U_{n i}\right)-f\left(t_{n}+c_{i} h\right)\right)-\delta_{n+1} .
\end{gathered}
$$

We will derive bounds for these errors.

4.2. Convergence of the exponential Euler method. For $s=1$, the only reasonable choice is the exponential form of Euler's method. Applied to (1.1), it is

$$
u_{n+1}=\mathrm{e}^{-h A} u_{n}+h \varphi_{1}(-h A) g\left(t_{n}, u_{n}\right) .
$$

For this method, we have the following convergence result.

TheOrem 4.2. Let the initial value problem (1.1) satisfy Assumptions 1-2 and consider for its numerical solution the exponential Euler method (4.15). Further assume that $f:[0, T] \rightarrow X$ is differentiable and that $\beta \in(0,1]$ is such that $\widetilde{A}^{\beta-1} f^{\prime} \in L^{\infty}(0, T ; V)$. Then, the following error bound holds

$$
\left\|u_{n}-u\left(t_{n}\right)\right\|_{V} \leq C \cdot h \sup _{0 \leq t \leq t_{n}}\left\|\widetilde{A}^{\beta-1} f^{\prime}(t)\right\|_{V}
$$

uniformly in $0 \leq n h \leq T$. The constant $C$ depends on $T$, but it is independent of $n$ and $h$.

Proof. The exponential Euler method satisfies the error recursion

$$
e_{n+1}=\mathrm{e}^{-h A} e_{n}+h \varphi_{1}(-h A)\left(g\left(t_{n}, u_{n}\right)-f\left(t_{n}\right)\right)-\delta_{n+1}
$$


with defects $\delta_{n+1}=\delta_{n+1}^{[1]}$ given by (4.7b) and (4.11). Solving recursion (4.16) gives

$$
e_{n}=h \sum_{j=0}^{n-1} \mathrm{e}^{-(n-j-1) h A} \varphi_{1}(-h A)\left(g\left(t_{j}, u_{j}\right)-f\left(t_{j}\right)\right)-\sum_{j=0}^{n-1} \mathrm{e}^{-j h A} \delta_{n-j} .
$$

Using (3.2a), Assumption 2 and Lemma 4.1, we may estimate this in $V$ by

$$
\left\|e_{n}\right\|_{V} \leq C h \sum_{j=0}^{n-2} t_{n-j-1}^{-\alpha}\left\|e_{j}\right\|_{V}+C h^{1-\alpha}\left\|e_{n-1}\right\|_{V}+C h \sup _{0 \leq t \leq t_{n}}\left\|\widetilde{A}^{\beta-1} f^{\prime}(t)\right\|_{V} .
$$

The application of a discrete Gronwall lemma [10, Lemma 4] concludes the proof.

REMARK. The above theorem can also be deduced from [10], since the exponential Euler method is a collocation method with $s=1$ and $c_{1}=0$.

4.3. Convergence results for second-order methods. We first derive a bound for the errors of the internal stages. Due to (2.2) and (4.1), we always have $\psi_{1, j}=0$. From (4.9) and (4.14a), we thus get with the help of Assumption 2

$$
\left\|E_{n i}\right\|_{V} \leq C\left\|e_{n}\right\|_{V}+C h^{1-\alpha} \max _{2 \leq j \leq i-1}\left\|E_{n j}\right\|_{V}+\left\|\Delta_{n i}^{[1]}\right\|_{V} .
$$

Applying Lemma 4.1 then shows the a priory bound

$$
\left\|E_{n i}\right\|_{V} \leq C\left\|e_{n}\right\|_{V}+C h^{1+\nu} \sup _{0 \leq \tau \leq 1}\left\|\widetilde{A}^{\nu-1} f^{\prime}\left(t_{n}+\tau h\right)\right\|_{V} .
$$

For second-order methods, we will satisfy the order conditions

$$
\psi_{1}(-h A)=0, \quad \psi_{2}(-h A)=0
$$

and take $q=2$ in (4.11). We are now in the position to state the convergence theorem.

TheOREM 4.3. Let the initial value problem (1.1) satisfy Assumptions 1 and 2 and consider for its solution an exponential Runge-Kutta method (2.1) satisfying (4.1), (4.2) and (4.18). Further assume that $f:[0, T] \rightarrow X$ is twice differentiable and that $\beta, \kappa \in(0,1]$ are such that $\widetilde{A}^{\beta-1} f^{\prime} \in L^{\infty}(0, T ; V)$ and $\widetilde{A}^{\kappa-1} f^{\prime \prime} \in L^{\infty}(0, T ; V)$. Then, the following error bound holds

$$
\left\|u_{n}-u\left(t_{n}\right)\right\|_{V} \leq C \cdot h^{1+\beta} \sup _{0 \leq t \leq t_{n}}\left\|\widetilde{A}^{\beta-1} f^{\prime}(t)\right\|_{V}+C \cdot h^{2} \sup _{0 \leq t \leq t_{n}}\left\|\widetilde{A}^{\kappa-1} f^{\prime \prime}(t)\right\|_{V}
$$

uniformly in $0 \leq n h \leq T$. The constant $C$ depends on $T$, but it is independent of $n$ and $h$.

REMARK. If $f^{\prime}, f^{\prime \prime} \in L^{\infty}(0, T ; X)$, the above theorem is applicable with $\beta=\kappa=$ $1-\alpha$. For $\beta=1$, the theorem yields order 2 . Under the slightly weaker regularity assumptions $f^{\prime}(0) \in V$ and $f^{\prime \prime} \in L^{1}(0, T ; V)$, we obtain an alternative second-order error bound

$$
\left\|u_{n}-u\left(t_{n}\right)\right\|_{V} \leq C \cdot h^{2}\left(\left\|f^{\prime}(0)\right\|_{V}+\int_{0}^{t_{n}}\left\|f^{\prime \prime}(\tau)\right\|_{V} \mathrm{~d} \tau\right) .
$$

This follows easily from the proof of Theorem 4.3. 
Proof. Solving the error recursion (4.14b) in the same way as in the proof of Theorem 4.2 gives

$$
\left\|e_{n}\right\|_{V} \leq C h^{1-\alpha}\left\|E_{n-1}\right\|_{V}+C h \sum_{j=0}^{n-2} t_{n-j-1}^{-\alpha}\left\|E_{j}\right\|_{V}+\left\|\sum_{j=0}^{n-1} \mathrm{e}^{-j h A} \delta_{n-j}\right\|_{V}
$$

with $\left\|E_{j}\right\|_{V}=\max _{2 \leq k \leq s}\left\|E_{j k}\right\|_{V}$. After inserting the bounds (4.17) and (4.13b), the proof is concluded by applying a discrete Gronwall lemma.

If $g:[0, T] \times V \rightarrow X$ is twice differentiable, its derivatives

$$
J_{n}=\frac{\partial g}{\partial u}\left(t_{n}, u\left(t_{n}\right)\right), \quad K_{n}=\frac{\partial^{2} g}{\partial t \partial u}\left(t_{n}, u\left(t_{n}\right)\right)
$$

are bounded operators from $V$ to $X$.

Lemma 4.4. Under Assumption 3, we have

$$
\begin{aligned}
g\left(t_{n}+c_{i} h, U_{n i}\right)-f\left(t_{n}+c_{i} h\right) & =J_{n} E_{n i}+c_{i} h K_{n} E_{n i}+Q_{n i} \\
g\left(t_{n}, u_{n}\right)-f\left(t_{n}\right) & =J_{n} e_{n}+Q_{n}
\end{aligned}
$$

with remainders $Q_{n i}$ and $Q_{n}$ satisfying the bounds

$$
\left\|Q_{n i}\right\| \leq C \cdot\left(h^{2}+\left\|E_{n i}\right\|_{V}\right)\left\|E_{n i}\right\|_{V}, \quad\left\|Q_{n}\right\| \leq C \cdot\left\|e_{n}\right\|_{V}^{2},
$$

as long as the errors $E_{n i}$ and $e_{n}$ remain in a sufficiently small neighborhood of 0.

Proof. Using Taylor series expansion, we get

$$
\begin{aligned}
g\left(t_{n}+c_{i} h, U_{n i}\right) & -f\left(t_{n}+c_{i} h\right)=\frac{\partial g}{\partial u}\left(t_{n}+c_{i} h, u\left(t_{n}+c_{i} h\right)\right) E_{n i} \\
& +\int_{0}^{1}(1-\tau) \frac{\partial^{2} g}{\partial u^{2}}\left(t_{n}+c_{i} h, u\left(t_{n}+c_{i} h\right)+\tau E_{n i}\right)\left(E_{n i}, E_{n i}\right) \mathrm{d} \tau .
\end{aligned}
$$

Expanding the first term on the right-hand side at $t_{n}$ yields the desired result. $\square$

Inserting (4.19a) into the recursion (4.14b) shows that the main contribution of the defects to the global error is given by the term

$$
h \sum_{j=0}^{n-1} \mathrm{e}^{-j h A} \sum_{i=1}^{s} b_{i}(-h A) J_{n} \Delta_{n-j-1, i}^{[1]} .
$$

Let $\gamma<1$ and $0 \leq \mu \leq 1-\beta$ be such that

$$
\left\|\widetilde{A}^{-\gamma} J_{n} \widetilde{A}^{\mu}\right\|_{V \leftarrow V} \leq C .
$$

This estimate trivially holds for $\gamma=\alpha$ and $\mu=0$. But there are more favorable situations in which $\mu>0$. Then, the expression (4.20) is bounded by

$$
C \cdot h^{1+\beta+\mu} \sup _{0 \leq t \leq t_{n}}\left\|\widetilde{A}^{\beta-1} f^{\prime}(t)\right\|_{V}
$$

which slightly improves the error bound of the theorem. We do not elaborate this point here further. 
4.4. Convergence results for higher-order methods. An explicit expansion of $E_{n i}$ in terms of $\Delta_{n i}$ and $e_{n}$ is easily obtained with the help of Lemma 4.4 by inserting the expressions of the lemma recursively into (4.14a). Depending on the size of $s$ and $\alpha$, however, this expansion will consist of a great number of terms that all give rise to order conditions for the method. For example, the arising term

$$
h^{\ell+2} \sum_{j_{1}=1}^{i-1} a_{i j_{1}}(-h A) J_{n} \cdots \sum_{j_{\ell}=1}^{j_{\ell-1}} a_{j_{\ell-1} j_{\ell}}(-h A) J_{n} \cdot \psi_{2, j_{\ell}}(-h A) f^{\prime}\left(t_{n}\right)
$$

has classical order $\ell+2$ and can be neglected for $\ell \geq 2$ in a fourth-order method. In our context, however, it has only order $1+(\ell+1)(1-\alpha)$, and this might be a small number, even for large $\ell$. In that case, the term cannot be longer neglected.

To keep our presentation simple, we will consider in the remainder of this section the case $\alpha=0$ only. It is therefore no longer necessary to distinguish between the spaces $V$ and $X$.

LEMMA 4.5. Under the assumptions of Lemma 4.4 and $\alpha=0$, there exist bounded operators $\mathcal{N}_{n i}\left(e_{n}\right)$ on $X$ such that

$$
\begin{aligned}
E_{n i}=\mathcal{N}_{n i}\left(e_{n}\right) e_{n} & -h^{2} \psi_{2, i}(-h A) f^{\prime}\left(t_{n}\right)-h^{3} \psi_{3, i}(-h A) f^{\prime \prime}\left(t_{n}\right) \\
& -h^{3} \sum_{j=2}^{i-1} a_{i j}(-h A) J_{n} \psi_{2, j}(-h A) f^{\prime}\left(t_{n}\right)+h^{4} \mathcal{R}_{n i},
\end{aligned}
$$

with uniformly bounded remainders $\left\|\mathcal{R}_{n i}\right\| \leq C$.

Proof. The formula follows easily from recursion (4.14a), the representation of the defects (4.9), and from Lemma 4.4 by an induction argument.

An important consequence of the above lemma is the following representation of the errors $e_{n}$. It is the key result for obtaining the stiff order conditions.

LEMMA 4.6. Under the assumptions of Lemma 4.4 and $\alpha=0$, there exist uniformly bounded operators $\mathcal{N}_{n}\left(e_{n}\right)$ on $X$ such that the global errors $e_{n}$ satisfy the following recursion

$$
\begin{aligned}
e_{n+1}= & \mathrm{e}^{-h A} e_{n}+h \mathcal{N}_{n}\left(e_{n}\right) e_{n}-h^{2} \psi_{2}(-h A) f^{\prime}\left(t_{n}\right) \\
& -h^{3} \psi_{3}(-h A) f^{\prime \prime}\left(t_{n}\right)-h^{3} \sum_{i=1}^{s} b_{i}(-h A) J_{n} \psi_{2, i}(-h A) f^{\prime}\left(t_{n}\right) \\
& -h^{4} \psi_{4}(-h A) f^{\prime \prime \prime}\left(t_{n}\right)-h^{4} \sum_{i=1}^{s} b_{i}(-h A) J_{n} \psi_{3, i}(-h A) f^{\prime \prime}\left(t_{n}\right) \\
& -h^{4} \sum_{i=1}^{s} b_{i}(-h A) J_{n} \sum_{j=2}^{i-1} a_{i j}(-h A) J_{n} \psi_{2, j}(-h A) f^{\prime}\left(t_{n}\right) \\
& -h^{4} \sum_{i=1}^{s} b_{i}(-h A) c_{i} K_{n} \psi_{2, j}(-h A) f^{\prime}\left(t_{n}\right)+h^{5} \mathcal{R}_{n},
\end{aligned}
$$

with uniformly bounded remainders $\left\|\mathcal{R}_{n}\right\| \leq C$.

Proof. The formula follows easily from recursion $(4.14 \mathrm{~b})$, the representation of the defects (4.11), and Lemma 4.5.

The stiff order conditions can easily be identified in (4.24). For clarity, we have collected them in Table 4.1. With these preparations, we are now in the position to formulate a more general convergence result. 


\begin{tabular}{|c|c|l|}
\hline No. & order & order condition \\
\hline 1 & 1 & $\psi_{1}(-h A)=0$ \\
\hline 2 & 2 & $\psi_{2}(-h A)=0$ \\
3 & 2 & $\psi_{1, i}(-h A)=0$ \\
\hline 4 & 3 & $\psi_{3}(-h A)=0$ \\
5 & 3 & $\sum_{i=1}^{s} b_{i}(-h A) J \psi_{2, i}(-h A)=0$ \\
\hline 6 & 4 & $\psi_{4}(-h A)=0$ \\
7 & 4 & $\sum_{i=1}^{s} b_{i}(-h A) J \psi_{3, i}(-h A)=0$ \\
8 & 4 & $\sum_{i=1}^{s} b_{i}(-h A) J \sum_{j=2}^{i-1} a_{i j}(-h A) J \psi_{2, j}(-h A)=0$ \\
9 & 4 & $\sum_{i=1}^{s} b_{i}(-h A) c_{i} K \psi_{2, i}(-h A)=0$ \\
\hline
\end{tabular}

TABLE 4.1

Stiff order conditions for explicit exponential Runge-Kutta methods for $\alpha=0$. Here $J$ and $K$ denote arbitrary bounded operators on $X$. The functions $\psi_{i}$ and $\psi_{k, \ell}$ are defined in (4.12) and (4.10), respectively.

TheOREM 4.7. Let the initial value problem (1.1) satisfy Assumptions 1-3 with $\alpha=0$ and consider for its numerical solution an explicit exponential Runge-Kutta method (2.1) satisfying (4.1) and (4.2). For $2 \leq p \leq 4$, assume that the order conditions of Table 4.1 hold up to order $p-1$ and that $\psi_{p}(0)=0$. Further assume that the remaining conditions of order $p$ hold in a weaker form with $b_{i}(0)$ instead of $b_{i}(-h A)$ for $2 \leq i \leq s$. Then, the numerical solution $u_{n}$ satisfies the error bound

$$
\left\|u_{n}-u\left(t_{n}\right)\right\| \leq C \cdot h^{p},
$$

uniformly in $0 \leq n h \leq T$. The constant $C$ depends on $T$, but it is independent of $n$ and $h$.

Proof. Inserting the order conditions into the recursion of Lemma 4.6 yields

$$
e_{n+1}=\mathrm{e}^{-h A} e_{n}+h \mathcal{N}_{n}\left(e_{n}\right) e_{n}+h^{p} \mathcal{T}_{n}+h^{p+1} \mathcal{R}_{n}
$$

with bounded remainders $\mathcal{R}_{n}$ depending on $p$. Here, $\mathcal{T}_{n}$ denote the terms multiplying $h^{p}$ in Lemma 4.6. Solving the above error recursion gives

$$
e_{n}=h \sum_{j=0}^{n-1} \mathrm{e}^{-(n-j) h A} \mathcal{N}_{j}\left(e_{j}\right) e_{j}+h^{p} \sum_{j=0}^{n-1} \mathrm{e}^{-j h A} \mathcal{T}_{n-j-1}+h^{p+1} \sum_{j=0}^{n-1} \mathrm{e}^{-j h A} \mathcal{R}_{n-j-1}
$$

In order to bound the second term on the right-hand side, we use the assumptions on the conditions of order $p$ and apply Lemma 4.8 below. Using further the stability 
estimate (3.2a) and the bounds for $\mathcal{R}_{j}$ and for $\mathcal{N}_{j}\left(e_{j}\right)$ finally yields

$$
\left\|e_{n}\right\| \leq C h \sum_{j=0}^{n-1}\left\|e_{j}\right\|+C h^{p}
$$

Thus, an application of the classical Gronwall lemma concludes the proof.

The following lemma was used in the above proof.

Lemma 4.8. Under the above assumptions, let $\varrho_{i}:[0, T] \rightarrow X$ for $1 \leq i \leq s$ denote differentiable functions with bounded derivatives. Then

$$
\left\|\sum_{j=0}^{n-1} \mathrm{e}^{-j h A} \sum_{i=1}^{s}\left(b_{i}(-h A)-b_{i}(0)\right) \varrho_{i}\left(t_{n-j-1}\right)\right\| \leq C .
$$

Proof. We first note that there exists bounded operators $\widetilde{b}_{i}(-h A)$ with

$$
b_{i}(-h A)-b_{i}(0)=h A \cdot \widetilde{b}_{i}(-h A)
$$

The bound (4.25) now follows at once from Lemma 3.2 with $w_{j}=\mathrm{e}^{-j h A} h A \cdot \widetilde{b}_{i}(-h A)$ and $v_{j}=\varrho_{i}\left(t_{j-1}\right)$ by using the stability bound $(3.2 \mathrm{~b})$.

REMARK. The proof of Theorem 4.7 does not extend immediately to variable step sizes. The reason for this lies in the use of the summation-by-parts formula (3.3). Assuming, however, that the method satisfies the conditions of order $p$ in the strong sense of Table 4.1, then Lemma 4.8 is no longer needed. In that case, the theorem obviously holds for variable step sizes, too.

4.5. Existence of explicit methods of arbitrarily high order. In our recent paper [10] we have shown that, under the above assumptions, an $s$-stage exponential Runge-Kutta method of collocation type with coefficients $\widehat{a}_{i j}(-h A)$ and $\widehat{b}_{i}(-h A)$ satisfies the error bound

$$
\left\|\widehat{u}_{n}-u\left(t_{n}\right)\right\|_{V} \leq C h^{s} \sup _{0 \leq t \leq T}\left\|f^{(s)}(t)\right\|
$$

uniformly on $0 \leq t_{n} \leq T$. It is further shown there that the equations for the internal stages can be solved by fixed-point iteration

$$
\widehat{U}_{n i}^{(k)}=\mathrm{e}^{-c_{i} h A} \widehat{u}_{n}+h \sum_{i=1}^{s} \widehat{a}_{i j}(-h A) g\left(t_{n}+c_{j} h, \widehat{U}_{n j}^{(k-1)}\right), \quad \widehat{U}_{n j}^{(0)}=\widehat{u}_{n} .
$$

For $\alpha=0$, we obviously gain one power of $h$ in each iteration. Performing $s$ iterations and setting $U_{n i}=\widehat{U}_{n i}^{(s)}, 1 \leq i \leq s$, and

$$
u_{n+1}=\mathrm{e}^{-h A} u_{n}+h \sum_{i=1}^{s} \widehat{b}_{i}(-h A) g\left(t_{n}+c_{j} h, U_{n j}\right)
$$

thus defines an explicit exponential Runge-Kutta method of order $s$ with $s^{2}$ stages. The construction shows that there exist explicit exponential Runge-Kutta methods of arbitrarily high order. For general $\alpha$, however, we gain only $h^{1-\alpha}$ in each iteration. Therefore, a lot of explicit stages might be necessary for obtaining order $s$. 
5. A discussion of explicit methods of orders up to four. In this section, we consider some examples of methods up to order four. Since the first-order exponential Euler method has already been analyzed in Section 4.2, we commence here with second-order methods. In the Butcher tableaus below, we use the abbreviations

$$
\varphi_{i, j}=\varphi_{i, j}(-h A)=\varphi_{i}\left(-c_{j} h A\right), \quad 2 \leq j \leq s .
$$

5.1. Second-order methods. Second-order methods require two internal stages at least. For two stages, the order conditions are

$$
\begin{aligned}
b_{1}(-h A)+b_{2}(-h A) & =\varphi_{1}(-h A), \\
b_{2}(-h A) c_{2} & =\varphi_{2}(-h A), \\
a_{21}(-h A) & =c_{2} \varphi_{1}\left(-c_{2} h A\right) .
\end{aligned}
$$

A straightforward elimination leads to the following one-parameter family of exponential Runge-Kutta methods

$$
\begin{array}{c|cc}
0 & & \\
c_{2} & c_{2} \varphi_{1,2} & \\
\hline & \varphi_{1}-\frac{1}{c_{2}} \varphi_{2} & \frac{1}{c_{2}} \varphi_{2}
\end{array}
$$

The coefficients are displayed as usual in a Butcher tableau. It is also possible to omit the function $\varphi_{2}$ by weakening condition $(5.2 \mathrm{~b})$ to $b_{2}(0) c_{2}=\varphi_{2}(0)=\frac{1}{2}$. This yields another one-parameter family of methods

$$
\begin{array}{c|cc}
0 & \\
c_{2} & \multicolumn{2}{|c}{c_{2} \varphi_{1,2}} \\
\hline & \left(1-\frac{1}{2 c_{2}}\right) \varphi_{1} & \frac{1}{2 c_{2}} \varphi_{1}
\end{array}
$$

Note that the choice $c_{2}=\frac{1}{2}$ yields $b_{1}=0$.

Methods (5.3) and (5.4) have been proposed already by Strehmel and Weiner [25, Example 4.2.2] in the context of adaptive Runge-Kutta methods, where the functions $\varphi_{j}$ are usually approximated by certain rational functions. It is shown in $[25$, Section 4.5.3] that both methods are B-consistent of order one.

Method (5.3) satisfies the assumptions of Theorem 4.3 and thus converges with order $1+\beta$, in general. Method (5.4) can be analyzed in a similar way, as it differs from (5.3) in the choice of the quadrature rule for $u_{n+1}$ only. The defects at the grid points have now the form

$$
\begin{aligned}
\delta_{n+1}=\frac{h^{2}}{2}\left(2 \varphi_{2}(-h A)\right. & \left.-\varphi_{1}(-h A)\right) f^{\prime}\left(t_{n}+\frac{h}{2}\right) \\
& +\int_{0}^{h} \mathrm{e}^{-(h-\tau) A} \int_{h / 2}^{\tau}(\tau-\xi) f^{\prime \prime}\left(t_{n}+\xi\right) \mathrm{d} \xi .
\end{aligned}
$$

Since $2 \varphi_{2}(0)-\varphi_{1}(0)=0$, the first term of (5.5) contributes with

$$
C \cdot h^{1+\beta}\left(\left\|\widetilde{A}^{\beta-1} f^{\prime}(0)\right\|_{V}+\int_{0}^{t_{n}}\left\|\widetilde{A}^{\beta-1} f^{\prime \prime}(t)\right\|_{V} \mathrm{~d} t\right)
$$

to the global error. This is seen with the help of Lemma 3.2. Thus, Theorem 4.3 holds with the additional error term (5.6) for the modified method (5.4), too. In 
particular, we get a second-order error bound for $\beta=1$. Note, however, that (5.6) cannot be improved under a condition of the type (4.21). For situations in which $f^{\prime}(t), f^{\prime \prime}(t) \notin V$, method (5.3) is therefore preferable to method (5.4) which can be affected by an order reduction, see also Figure 6.3 below.

5.2. Third-order methods. We will continue with 3 -stage methods. In the following, we assume $\alpha=0$. The order conditions for 3-stage methods are

$$
\begin{aligned}
b_{1}(-h A)+b_{2}(-h A)+b_{3}(-h A) & =\varphi_{1}(-h A), \\
b_{2}(-h A) c_{2}+b_{3}(-h A) c_{3} & =\varphi_{2}(-h A), \\
a_{21}(-h A) & =c_{2} \varphi_{1}\left(-c_{2} h A\right), \\
a_{31}(-h A)+a_{32}(-h A) & =c_{3} \varphi_{1}\left(-c_{3} h A\right), \\
b_{2}(-h A) c_{2}^{2}+b_{3}(-h A) c_{3}^{2} & =2 \varphi_{3}(-h A), \\
b_{2}(-h A) J c_{2}^{2} \varphi_{2}\left(-c_{2} h A\right)+b_{3}(-h A) J \psi_{2,3} & =0,
\end{aligned}
$$

where

$$
\psi_{2,3}=c_{3}^{2} \varphi_{2}\left(-c_{3} h A\right)-c_{2} a_{32}(-h A) .
$$

Condition (5.7f) can be satisfied by $b_{2}=0$ and $\psi_{2,3}=0$ or $b_{2}=\gamma b_{3}$ and $c_{2}^{2} \varphi_{2,2}+$ $\gamma \psi_{2,3}=0$. However, both choices contradict conditions (5.7b) and (5.7e). We thus weaken $(5.7 \mathrm{e})$ to

$$
b_{2}(0) c_{2}^{2}+b_{3}(0) c_{3}^{2}=2 \varphi_{3}(0)=1 / 3 .
$$

The choice $b_{2}=0$ leads to the following one-parameter family of third-order methods

$$
\begin{array}{c|ccc}
0 & & & \\
c_{2} & c_{2} \varphi_{1,2} & & \\
\frac{2}{3} & \frac{2}{3} \varphi_{1,3}-\frac{4}{9 c_{2}} \varphi_{2,3} & \frac{4}{9 c_{2}} \varphi_{2,3} & \\
\hline & \varphi_{1}-\frac{3}{2} \varphi_{2} & 0 & \frac{3}{2} \varphi_{2}
\end{array}
$$

The other choice $b_{2}=\gamma b_{3}$ leads to the two-parameter family of methods of order 3

$$
\begin{array}{c|ccc}
0 & & & \\
c_{2} & c_{2} \varphi_{1,2} & & \\
c_{3} & c_{3} \varphi_{1,3}-a_{32} & \gamma c_{2} \varphi_{2,2}+\frac{c_{3}^{2}}{c_{2}} \varphi_{23} & \\
\hline & \varphi_{1}-b_{2}-b_{3} & \frac{\gamma}{\gamma c_{2}+c_{3}} \varphi_{2} & \frac{1}{\gamma c_{2}+c_{3}} \varphi_{2}
\end{array}
$$

where $\gamma, c_{2}$ and $c_{3}$ have to satisfy the restriction

$$
2\left(\gamma c_{2}+c_{3}\right)=3\left(\gamma c_{2}^{2}+c_{3}^{2}\right) .
$$

Another possibility is to weaken (5.7f) to

$$
b_{2}(0) c_{2}^{2} \varphi_{2}\left(-c_{2} h A\right)+b_{3}(0)\left(c_{3}^{2} \varphi_{2}\left(-c_{3} h A\right)-c_{2} a_{32}(-h A)\right)=0 .
$$

The order conditions of the underlying Runge-Kutta method of order 3 show that

$$
b_{2}(0)=\frac{2-3 c_{3}}{6 c_{2}\left(c_{2}-c_{3}\right)}, \quad b_{3}(0)=\frac{2-3 c_{2}}{6 c_{3}\left(c_{3}-c_{2}\right)},
$$


see [7, Exercise II.1.4]. Here, we have to choose $c_{2} \neq 2 / 3$ and $c_{2} \neq c_{3}$ except for $c_{2}=c_{3}=2 / 3$ where $b_{2}(0)+b_{3}(0)=3 / 4$.

A three-parameter family of methods that involve $\varphi_{1}$ and $\varphi_{2}$ only is given by

$$
\begin{array}{c|ccc}
0 & & & \\
c_{2} & c_{2} \varphi_{1,2} & & \\
c_{3} & c_{3} \varphi_{1,3}-a_{32} & \frac{c_{3}^{2}}{c_{2}} \varphi_{2,3}-\frac{2-3 c_{3}}{2-3 c_{2}} c_{3} \varphi_{2,2} & \\
\hline & \varphi_{1}-b_{2}-b_{3} & \alpha_{2} \varphi_{1}+\beta_{2} \varphi_{2} & \alpha_{3} \varphi_{1}+\beta_{3} \varphi_{2}
\end{array}
$$

In addition to $c_{2}$ and $c_{3}$, one of the coefficients $\alpha_{i}, \beta_{i}$ can be chosen arbitrarily and the remaining ones are determined by the linear system

$$
c_{2} \alpha_{2}+c_{3} \alpha_{3}=0, \quad \alpha_{3}+\frac{1}{2} \beta_{3}=b_{3}(0), \quad c_{2} \beta_{2}+c_{3} \beta_{3}=1 .
$$

Note that for $\alpha_{2}=0$ and $\beta_{2}=\gamma \beta_{3}$ we recover condition (5.10) and obtain the scheme (5.9). Setting $\alpha_{2}=\beta_{2}=0$ leads to $b_{2}(-h A)=0$ and the condition $c_{3}=2 / 3$, which results in the scheme (5.8).

We next discuss some related methods which can be found in the literature.

Strehmel and Weiner [25, Example 4.5.4] proved that for a 3-stage adaptive Runge-Kutta method with second-order B-consistency, the condition $b_{2}=0$ is necessary. They proposed the following family of such methods

$$
\begin{array}{c|ccc}
0 & & & \\
c_{2} & c_{2} \varphi_{1,2} & & \\
1 & \varphi_{1,3}-\frac{1}{c_{2}} \varphi_{2,3} & \frac{1}{c_{2}} \varphi_{2,3} & \\
\hline & \varphi_{1}-\varphi_{2} & 0 & \varphi_{2}
\end{array}
$$

These methods satisfy conditions $(5.7 \mathrm{a}),(5.7 \mathrm{~b}),(5.7 \mathrm{c})$, and $(5.7 \mathrm{~d})$, i.e. all conditions of Table 4.1 up to order 2. However, the conditions (5.7f) and (5.7g) of order 3 are not satisfied, not even in a weak form. Therefore, these methods are of second-order only. Since they are more expensive than the 2-stage methods proposed in the previous section, the latter should be preferred for semilinear parabolic problems.

Cox and Matthews [3] constructed a method called ETD3RK which reads

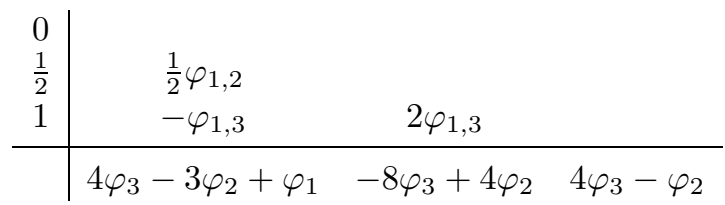

This method uses $\varphi_{3}$ and satisfies conditions (5.7a), (5.7b), (5.7c), (5.7d), and (5.7g). However, condition (5.7f) is only satisfied in a very weak form (where all arguments are evaluated for $A=0$ ). This leads to an order reduction to order two in the worst case. The same conditions are satisfied by ETD2RK3 from [3].

The method ETD2CF3 is a variant of the commutator-free Lie group method CF3 due to Celledoni, Marthinsen and Owren [2]. It is given by

$$
\begin{array}{c|ccc}
0 & & & \\
\frac{1}{3} & \frac{1}{3} \varphi_{1,2} & & \\
\frac{2}{3} & \frac{2}{3} \varphi_{1,3}-\frac{4}{3} \varphi_{2,3} & \frac{4}{3} \varphi_{2,3} & \\
\hline & \varphi_{1}-\frac{9}{2} \varphi_{2}+9 \varphi_{3} & 6 \varphi_{2}-18 \varphi_{3} & -\frac{3}{2} \varphi_{2}+9 \varphi_{3} \\
& & 16 &
\end{array}
$$


This method satisfies (5.7a)-(5.7c) and weak forms of (5.7d) and (5.7f), where $b_{i}(-h A)$ is evaluated for $A=0$. Therefore this method is of order three for $\alpha=0$.

5.3. Fourth-order methods. Again we assume $\alpha=0$. The order conditions for $s$-stage methods up to order four are

$$
\begin{gathered}
\sum_{i=1}^{s} b_{i}(-h A)=\varphi_{1}(-h A), \\
\sum_{i=2}^{s} b_{i}(-h A) c_{i}=\varphi_{2}(-h A), \\
\sum_{j=1}^{i-1} a_{i j}(-h A)=c_{i} \varphi_{1}\left(-c_{i} h A\right), \\
\sum_{i=2}^{s} b_{i}(-h A) c_{i}^{2}=2 \varphi_{3}(-h A), \\
\sum_{i=2}^{s} b_{i}(-h A) J\left(\varphi_{2}\left(-c_{i} h A\right) c_{i}^{2}-\sum_{j=2}^{i-1} a_{i j}(-h A) c_{j}\right)=0, \\
\sum_{i=2}^{s} b_{i}(-h A) c_{i}^{3}=6 \varphi_{4}(-h A), \\
\sum_{i=2}^{s} b_{i}(-h A) J\left(\varphi_{3}\left(-c_{i} h A\right) c_{i}^{3}-\frac{1}{2} \sum_{j=2}^{i-1} a_{i j}(-h A) c_{j}^{2}\right)=0 \\
\sum_{j=2}^{s} a_{i j}(-h A) J\left(\varphi_{2}\left(-c_{j} h A\right) c_{j}^{2}-\sum_{k=2}^{j-1} a_{j k}(-h A) c_{k}\right)=0, \\
\left.\sum_{i=1}^{i-1} a_{i j}(-h A) c_{j}\right)=0 .
\end{gathered}
$$

From $(5.14 \mathrm{a}),(5.14 \mathrm{~b})$, and $(5.14 \mathrm{~d})$ we deduce that any fourth-order method has to involve $\varphi_{1}, \varphi_{2}$, and $\varphi_{3}$. By Theorem 4.7 it is sufficient to satisfy condition 6 of Table 4.1 in the weakened form $\psi_{4}(0)=0$, i.e. to replace $(5.14 \mathrm{f})$ by

$$
\sum_{i=2}^{s} b_{i}(0) c_{i}^{3}=\frac{1}{4} .
$$

Cox and Matthews [3] proposed the following exponential variant of the classical Runge-Kutta method

$$
\begin{array}{c|cccc}
0 & & & \\
\frac{1}{2} & \frac{1}{2} \varphi_{1,2} & & \\
\frac{1}{2} & 0 & \frac{1}{2} \varphi_{1,3} & & \\
1 & \frac{1}{2} \varphi_{1,3}\left(\varphi_{0,3}-1\right) & 0 & \varphi_{1,3} & \\
\hline & \varphi_{1}-3 \varphi_{2}+4 \varphi_{3} & 2 \varphi_{2}-4 \varphi_{3} & 2 \varphi_{2}-4 \varphi_{3} & 4 \varphi_{3}-\varphi_{2}
\end{array}
$$

This method satisfies conditions $1-4$ of Table 4.1 , the weakened but sufficient condition $6\left(\psi_{4}(0)=0\right)$, but not conditions $5,7,8$ and 9 . However, it satisfies a weakened 
form of conditions 5 and 9 (because $\psi_{2,2}(0)+\psi_{2,3}(0)=0$ and $\psi_{2,4}(0)=0$ ), and a very weak form of conditions 7 and 8 (where all arguments are evaluated for $A=0$ ). In the worst case, this leads to an order reduction to order two only.

Krogstad's method [12] for (1.1) is given by

$$
\begin{array}{c|cccc}
0 & & & \\
\frac{1}{2} & \frac{1}{2} \varphi_{1,2} & & & \\
\frac{1}{2} & \frac{1}{2} \varphi_{1,3}-\varphi_{2,3} & \varphi_{2,3} & & \\
1 & \varphi_{1,4}-2 \varphi_{2,4} & 0 & 2 \varphi_{2,4} & \\
\hline & \varphi_{1}-3 \varphi_{2}+4 \varphi_{3} & 2 \varphi_{2}-4 \varphi_{3} & 2 \varphi_{2}-4 \varphi_{3} & -\varphi_{2}+4 \varphi_{3}
\end{array}
$$

This method satisfies conditions $1-5$ and 9 of Table 4.1, the weakened but sufficient condition $6\left(\psi_{4}(0)=0\right)$, but not conditions 7 and 8 , which are only satisfied in a very weak form (where all arguments are evaluated for $A=0$ ). In the worst case, this leads to an order reduction to order three.

Strehmel and Weiner's method [25, Example 4.5.5] can be written as

$$
\begin{array}{c|cccc}
0 & & & & \\
\frac{1}{2} & \frac{1}{2} \varphi_{1,2} & & & \\
\frac{1}{2} & \frac{1}{2} \varphi_{1,3}-\frac{1}{2} \varphi_{2,3} & \frac{1}{2} \varphi_{2,3} & & \\
1 & \varphi_{1,4}-2 \varphi_{2,4} & -2 \varphi_{2,4} & 4 \varphi_{2,4} & \\
\hline & \varphi_{1}-3 \varphi_{2}+4 \varphi_{3} & 0 & 4 \varphi_{2}-8 \varphi_{3} & -\varphi_{2}+4 \varphi_{3}
\end{array}
$$

This method satisfies the conditions of Table 4.1 in exactly the same way as Krogstad's method. It thus converges in our situation with order three in the worst case. Strehmel and Weiner proved that the method is B-consistent of order two.

REMARK. Under favorable circumstances, each of the above methods can show a higher order of convergence (generically up to order 4). We will shortly discuss a typical situation when this happens. For instance, the method of Cox and Matthews satisfies condition 5 of Table 4.1 only in the very weak form

$$
\sum_{i=1}^{s} b_{i}(0) J_{n} \psi_{2, i}(0)=0 .
$$

According to Theorem 4.7, this may result in an order reduction down to order two. The term corresponding to condition 5 contributes to the global error via

$$
\begin{aligned}
& h^{3} \sum_{j=0}^{n-1} \mathrm{e}^{-(n-j-1) h A} \sum_{i=1}^{s} b_{i}(-h A) J_{j} \psi_{2, i}(-h A) f^{\prime \prime}\left(t_{j}\right)= \\
& \quad h^{3} \sum_{j=0}^{n-1} \mathrm{e}^{-(n-j-1) h A} \sum_{i=1}^{s}\left(b_{i}(-h A)-b_{i}(0)\right) J_{j} \psi_{2, i}(0) f^{\prime \prime}\left(t_{j}\right) \\
& +h^{3} \sum_{j=0}^{n-1} \mathrm{e}^{-(n-j-1) h A} \sum_{i=1}^{s} b_{i}(-h A) J_{j}\left(\psi_{2, i}(-h A)-\psi_{2, i}(0)\right) f^{\prime \prime}\left(t_{j}\right)
\end{aligned}
$$

The first part (5.18a) has order 3 by Lemma 4.8. In order to improve the second term, we assume that the operators $A$ and $J_{j}$ are such that $A^{-1} J_{j} \widetilde{A}^{\mu}$ is bounded on 
$X$ for some $0 \leq \mu \leq 1$. Then, due to

$$
\begin{aligned}
& \left\|A^{-1} J_{j}\left(\psi_{2, i}(-h A)-\psi_{2, i}(0)\right)\right\|_{X \leftarrow X}= \\
& \left\|A^{-1} J_{j}(h \widetilde{A})^{\mu} \cdot(h \widetilde{A})^{-\mu}\left(\psi_{2, i}(-h A)-\psi_{2, i}(0)\right)\right\|_{X \leftarrow X} \leq C h^{\mu},
\end{aligned}
$$

one gains (by applying once more Lemma 4.8) an additional order $\mu$ in the term (5.18b). Exactly this happens in the numerical examples of Section 6.

One might ask whether it is possible to modify the above methods in such a way that they have order 4 for semilinear parabolic problems. In fact this cannot be done without adding further stages, which is seen as follows: Assume $s=4, c_{2}=c_{3}=1 / 2$, and $c_{4}=1$. Due to the order conditions of the underlying method, we have $b_{4}(0) \neq 0$ and $\left|b_{2}(0)\right|+\left|b_{3}(0)\right|>0$. Condition 5 of Table 4.1 immediately yields $\psi_{2,4}=0$. Moreover, $b_{3} \neq 0$ since $\psi_{2,2} \neq 0$. Hence, condition 5 can be satisfied only if $b_{2}=\gamma b_{3}$. This leads to $\gamma \psi_{2,2}+\psi_{2,3}=0$, which gives $a_{32}=(1+\gamma) \frac{1}{2} \varphi_{2,3}$. This choice of $a_{32}$ contradicts condition 8 even in the weakened form, where $b_{i}(-h A)$ is replaced by $b_{i}(0)$.

Thus we consider the case $s=5$ and add the node $c_{5}=1 / 2$. In order to avoid the difficulty encountered above, we have to choose $b_{2}=b_{3}=0$. This requires $b_{4} b_{5} \neq 0$ and therefore we have to enforce $\psi_{2,4}=\psi_{2,5}=0$ by condition 5 for a method of order 3 . Therefore condition 9 is satisfied automatically. Condition 8 shows that $a_{42}=\gamma a_{43}$ and $a_{52}=\gamma a_{53}$. For simplicity, we choose $\gamma=1$. This gives $\psi_{2,2}+\psi_{2,3}=0$, which leads to $a_{32}=\varphi_{2,3}$. Unfortunately, condition 7 cannot be satisfied in a strong form, because $\psi_{3,4}=\psi_{3,5}=0$ contradicts $\psi_{2,4}=\psi_{2,5}=0$. Hence we only require the weak form with $b_{i}(0)$ instead of $b_{i}(-h A)$. This yields the following fourth-order scheme

$$
\begin{array}{c|ccccc}
0 & & & & & \\
\frac{1}{2} & \frac{1}{2} \varphi_{1,2} & & & & \\
\frac{1}{2} & \frac{1}{2} \varphi_{1,3}-\varphi_{2,3} & \varphi_{2,3} & & & \\
1 & \varphi_{1,4}-2 \varphi_{2,4} & \varphi_{2,4} & \varphi_{2,4} & & \\
\frac{1}{2} & \frac{1}{2} \varphi_{1,5}-2 a_{5,2}-a_{5,4} & a_{5,2} & a_{5,2} & \frac{1}{4} \varphi_{2,5}-a_{5,2} & \\
\hline & \varphi_{1}-3 \varphi_{2}+4 \varphi_{3} & 0 & 0 & -\varphi_{2}+4 \varphi_{3} & 4 \varphi_{2}-8 \varphi_{3}
\end{array}
$$

with

$$
a_{5,2}=\frac{1}{2} \varphi_{2,5}-\varphi_{3,4}+\frac{1}{4} \varphi_{2,4}-\frac{1}{2} \varphi_{3,5} .
$$

6. Numerical experiments. In this section we present some numerical experiments in order to verify the sharpness of our error bounds.

As a first example we consider the semilinear parabolic problem

$$
\frac{\partial U}{\partial t}(x, t)-\frac{\partial^{2} U}{\partial x^{2}}(x, t)=\frac{1}{1+U(x, t)^{2}}+\Phi(x, t)
$$

for $x \in[0,1]$ and $t \in[0,1]$, subject to homogeneous Dirichlet boundary conditions. The source function $\Phi$ is chosen in such a way that the exact solution of the problem is $U(x, t)=x(1-x) \mathrm{e}^{t}$.

We discretize this problem in space by standard finite differences with 200 grid points. Due to our theory, we expect to see order two for the two variants (5.3) and (5.4) with $c_{2}=\frac{1}{2}$ of the exponential Runge method and order three for the two variants 
(5.8) and (5.9) of the exponential Heun method with $c_{2}=1 / 3$ and $\gamma=1.52$. Note that for this example $\left\|A^{-1} J A\right\|$ is bounded. This gives us order four for Krogstad's method and order three for the Cox and Matthew's method. All these orders are confirmed by the results illustrated in Figure 6.1, where the errors are measured in the maximum norm.
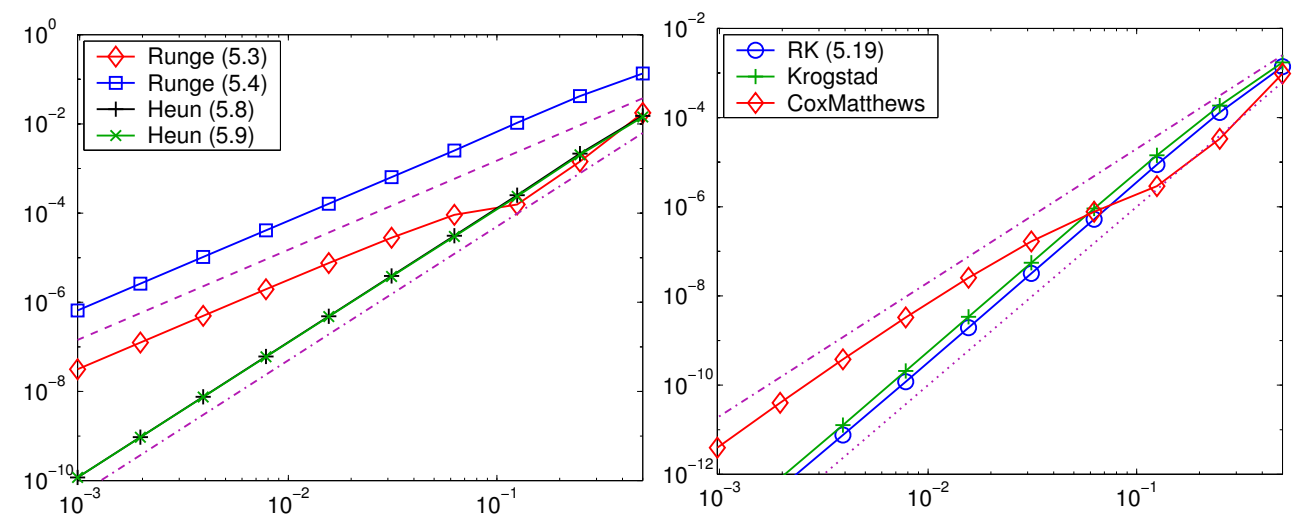

FIG. 6.1. The errors of various explicit exponential Runge-Kutta methods of orders two to four when applied to (6.1). The errors are measured in the maximum norm at $t=1$ and plotted as a function of the stepsize. For comparison, we added lines with slope two (dashed), three (dash-dotted), and four (dotted).

Next we consider the semilinear parabolic problem

$$
\frac{\partial U}{\partial t}(x, t)-\frac{\partial^{2} U}{\partial x^{2}}(x, t)=\int_{0}^{1} U(x, t) \mathrm{d} x+\Phi(x, t)
$$

for $x \in[0,1]$ and $t \in[0,1]$, subject to homogeneous Dirichlet boundary conditions. The source function $\Phi$ is again chosen such that $U(x, t)=x(1-x) \mathrm{e}^{t}$ is the exact solution.

We discretize this problem in space as in the first example, with the trapezoidal rule for the approximation of the integral. Again we can see order two for the two variants of the exponential Runge method and order three for the two variants of the exponential Heun method. However, since in this example only $\left\|A^{-1} J A^{1 / 2}\right\|$ is bounded, Krogstad's method and Cox and Matthew's method suffer from an order reduction. For Krogstad's method, we thus obtain order 3.5 while for the Cox-Matthews method we have order 2.5 only. The new exponential variant of the classical RungeKutta method is of full order 4 in this example.

In Figure 6.3 we present the errors of the two- and three-stage methods in the $V$-norm for the choice $X=L^{2}$ and $\alpha=1 / 2$. It can be seen that method (5.3) is of order two while method (5.4) is of order 1.75 only. The order reduction for (5.4) is perfectly explained by Theorem 4.3 with $\beta=3 / 4-\varepsilon$ for arbitrary $\varepsilon>0$, since the derivatives of $f$ are smooth functions that do not satisfy the boundary conditions. Method (5.3), however, has full order two, since condition (4.21) holds with $\gamma=1$ and $\mu=1 / 2$. See also our detailed discussion on this topic at the end of Section 4.3. By similar considerations it can be explained why the three-stage methods show order 2.75 instead of order three in this norm.

It is beyond the scope of this paper to discuss implementation details and to compare the efficiency of exponential methods with standard implicit schemes. Such 

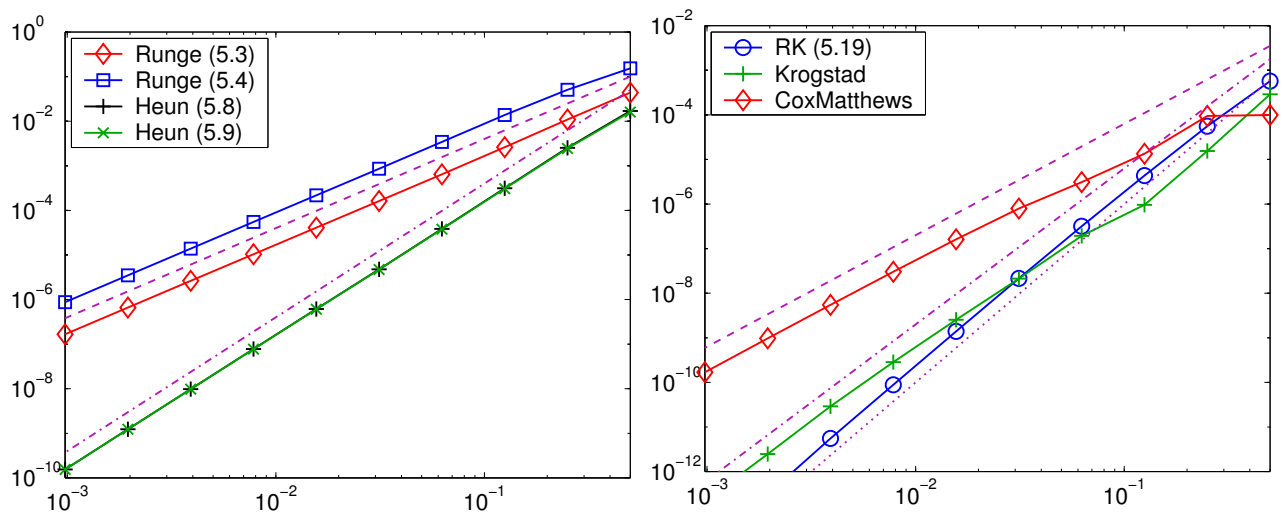

FIG. 6.2. The errors of various explicit exponential Runge-Kutta methods of orders two to four when applied to (6.2). The errors are measured in the maximum norm at $t=1$ and plotted as a function of the stepsize. For comparison, we added lines with different slopes. In the left figure, slope two is represented by a dashed line and slope three by a dash-dotted line. In the figure on the right, slope 2.5 is represented by a dashed line, slope 3.5 by a dash-dotted line, and slope 4 by a dotted line.

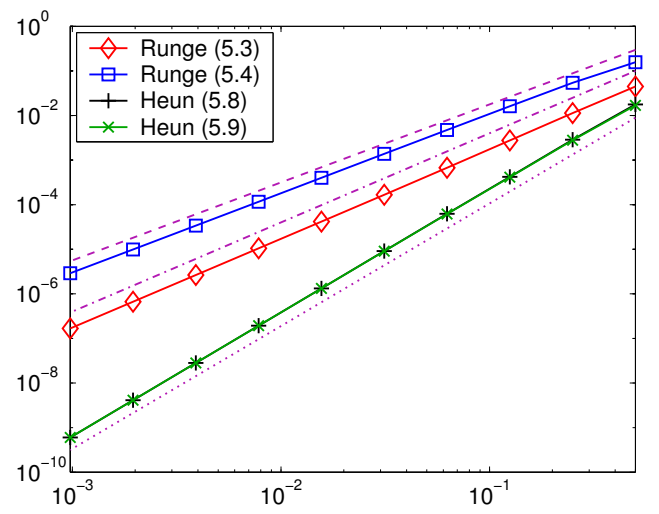

FIG. 6.3. The errors of various explicit exponential Runge-Kutta methods up to order three when applied to (6.2). The errors are measured at $t=1$ in a discrete $V$-norm for $X=L^{2}$ and $\alpha=1 / 2$. They are plotted as a function of the stepsize. For comparison, we added lines with different slopes: slope 1.75 is represented by a dashed line, slope 2 by a dash-dotted line, and slope 2.75 by a dotted line.

comparisons have been presented in $[3,11,12]$. Our aim here was to understand the convergence behavior of explicit methods and to present new order conditions which allow to construct methods up to order four in a systematic way.

Acknowledgments. The authors thank Ch. Lubich and J. Schweitzer for their continuous interest in our work and for inspiring discussions.

\section{REFERENCES}

[1] H. Berland, B. Owren, and B. Skaflestad, B-series and order conditions for exponential integrators, Tech. Report No. 5, Department of Mathematical Sciences, Norwegian University of Science and Technology Trondheim, 2004. 
[2] E. Celledoni, A. Marthinsen, And B. Owren, Commutator-free Lie group methods, Future Generation Computer Systems, 19 (2003), pp. 341-352.

[3] S. Cox And P. Matthews, Exponential time differencing for stiff systems, Journal of Computational Physics, 176 (2002), pp. 430-455.

[4] B. Ehle and J. LaWson, Generalized Runge-Kutta processes for stiff initial value problems, J. Inst. Math. Appl., 16 (1975), pp. 11-21.

[5] J. V. D. Eshof AND M. HochBruCK, Preconditioning Lanczos approximations to the matrix exponential, tech. report, Heinrich-Heine Universität Düsseldorf, 2004.

[6] A. FRIEDLI, Verallgemeinerte Runge-Kutta Verfahren zur Lösung steifer Differentialgleichungssysteme, in Numerical Treatment of Differential Equations, R. Burlirsch, R. Grigorieff, and J. Schröder, eds., vol. 631 of Lecture Notes in Mathematics, Springer, Berlin, 1978.

[7] E. Hairer, S. Nørsett, And G. Wanner, Solving Ordinary Differential Equations I-Nonstiff Problems, vol. 8 of Springer Series in Computational Mathematics, Springer-Verlag, Berlin Heidelberg, 2nd ed., 1991.

[8] D. Henry, Geometric Theory of Semilinear Parabolic Equations, vol. 840 of Lecture Notes in Mathematics, Springer, Berlin, Heidelberg, 1981.

[9] M. Hochbruck, C. Lubich, ANd H. Selhofer, Exponential integrators for large systems of differential equations, SIAM J. Sci. Comput., 19 (1998), pp. 1552-1574.

[10] M. Hochbruck AND A. Ostermann, Exponential Runge-Kutta methods for parabolic problems, Applied Numerical Mathematics, (to appear).

[11] A.-K. Kassam and L. Trefethen, Fourth-order time stepping for stiff PDEs, SIAM J. Sci. Comput., (to appear)

[12] S. Krogstad, Generalized integrating factor methods for stiff PDEs, J. Comput. Phys. (to appear)

[13] J. Lambert and S. Sigurdsson, Multistep methods with variable matrix coefficients, SIAM J. Numer. Anal., 9 (1972), pp. 715-733.

[14] J. Lawson, Generalized Runge-Kutta processes for stable systems with large Lipschitz constants, SIAM J. Numer. Anal., 4 (1967), pp. 372-380.

[15] C. Lubich and A. Ostermann, Linearly implicit time discretization of non-linear parabolic equations, IMA J. Numer. Anal., 15 (1995), pp. 555-583.

[16] _ Runge-Kutta approximation of quasi-linear parabolic equations, Math. Comp., 64 (1995), pp. 601-627.

[17] A. LunARDi, Analytic Semigroups and Optimal Regularity in Parabolic Problems, Birkhäuser, Basel, 1995.

[18] B. Minchev, Lie group integrators with nonautonomous frozen vector fields, Tech. Report 276, Department of Informatics, University of Bergen, 2004.

[19] C. Moler And C. VAn LoAn, Nineteen dubious ways to compute the exponential of a matrix, twenty-five years later, SIAM Rev., 45 (2003), pp. 3-49 (electronic).

[20] I. Moret And P. Novati, RD rational approximations of the matrix exponential, BIT, 44 (2004), pp. 595-615.

[21] S. NøRSETt, An A-stable modification of the Adams-Bashforth methods, in Conference on the Numerical Solution of Differential Equations, J. Morris, ed., vol. 109 of Lecture Notes in Mathematics, Springer, Berlin, 1969.

[22] A. PAZY, Semigroups of Linear Operators and Applications to Partial Differential Equations, Springer, New York, 1983.

[23] D. Pope, An exponential method of numerical integration of ordinary differential equations, Comm. ACM, 6 (1963), pp. 491-493.

[24] K. Strehmel and R. Weiner, B-convergence results for linearly implicit one step methods, BIT, 27 (1987), pp. 264-281.

[25] — Linear-implizite Runge-Kutta Methoden und ihre Anwendungen, Teubner, Leipzig, 1992.

[26] J. VERWER, On generalized linear multistep methods with zero-parasitic roots and an adaptive principal root, Numer. Math., 27 (1977), pp. 143-155. 